\title{
Small Fermi surface in the one-dimensional Kondo lattice model
}

\author{
J. C. Xavier, , E. Novais, \\ Instituto de Física Gleb Wataghin, Unicamp, Caixa Postal 6165, 13083-970 Campinas, São Paulo, Brazil
}

(Dated: 25th October 2018)

\begin{abstract}
We study the one-dimensional Kondo lattice model through the density matrix renormalization group (DMRG). Our results for the spin correlation function indicate the presence of a small Fermi surface in large portions of the phase diagram, in contrast to some previous studies that used the same technique. We argue that the discrepancy is due to the open boundary conditions, which introduce strong charge perturbations that strongly affect the spin Friedel oscillations.
\end{abstract}

PACS numbers: 75.10.-b, 71.10.Pm, 71.10.Hf

Several uncertainties still exist in our understanding of the physics of heavy fermion materials 1 The importance of solving these uncertainties has become even more pressing as we attempt to understand the anomalous behavior observed in the vicinity of the clean antiferromagnetic quantum critical point 2 The two major scenarios take radically different points of view. In the first one, conduction electrons are assumed to acquire their peculiar dynamics through an essentially perturbative coupling to the slpw critical modes of the antiferromagnetic background 3 Alternatively, the starting point is taken to be the strong coupling of the conduction electrons and the localized spins to form singlets, as in the single impurity Kondo problem. The nature of the quantum critical point is then linked to a non-trivial competition between the local dynamics apd the long wavelength antiferromagnetic fluctuations 3

One of the key assumptions of the second approach is the presence of a large Fermi surface (FS), namely one whose volume is given by including the localized spins in the count

$$
\frac{2 V_{F S}^{*}}{(2 \pi)^{D}}=n+1
$$

Behind this assumption lies a deep connection tween the Friedel sum rule of the single impurity Kondo problem 1 and Luttinger's theorem for the FS volume of a system of interacting fermions 8 The inclusion of the localized electron in the count is natural within an Anderson lattice model description at weak coupling but becomes doubtful at strong coupling where the Kondo lattice Hamiltonian is the effective low-energy theory. Hpwever, topologica arguments have been used, in one as well as in highen 10 dimensions, to show that indeed neutral gapless-excitations must exist at a $\mathbf{k}$-vector spanning a large FS.23 Furthermore, numerical studies of the onedimensional Kondo latticemodel have also pointed to the presence of a large FS11.12 In this paper, we show that a more careful analysis of the numerical evidence casts serious doubts on these conclusions and leaves open the question of the size of the Fermi surface in heavy fermion systems.

We considered the one-dimensional $S=\frac{1}{2}$ Kondo lat- tice Hamiltonian with $L$ sites

$$
H=-t \sum_{j=1, \sigma}^{L-1} c_{j, \sigma}^{\dagger} c_{j+1, \sigma}+J \sum_{j=1}^{L} \mathbf{S}_{j} \cdot \mathbf{s}_{j}
$$

where $c_{j \sigma}$ annihilates a conduction electron in site $j$ with spin projection $\sigma, \mathbf{S}_{j}$ is a localized spin $\frac{1}{2}$ operator and $\mathbf{s}_{j}=\frac{1}{2} \sum_{\alpha \beta} c_{j, \alpha}^{\dagger} \boldsymbol{\sigma}_{\alpha \beta} c_{j, \beta}$ is the conduction electron spin density operator. $J>0$ is the Kondo coupling constant between the conduction electrons and the local moments and the hopping amplitude $t$ is set to unity to fix the energy scale. We treated the model with the donsity matrix renormalization group (DMRG) technique 13,41 with open boundary conditions. We used the finite-size algorithm for sizes up to $L=120$ keeping up to $m=400$ states per block. The discarded weight was typically about $10^{-5}-10^{-8}$ in the final sweep.

There is compelling evidence that the onedimensional Kondo lattice model away from half-filling is a Tomonaga-Luttinger (TL) liquid 16 TL liquids with periodic boundary conditions, have charge and spin correlation functions given asymptotically by

$$
\begin{aligned}
\langle\delta n(0) \delta n(x)\rangle & =\frac{K_{\rho}}{(\pi x)^{2}}+A_{1} \frac{\cos \left(2 k_{F} x\right)}{x^{K_{\rho}+1}} \\
& +A_{2} \frac{\cos \left(4 k_{F} x\right)}{x^{4 K_{\rho}}} \\
\langle\mathbf{S}(0) \cdot \mathbf{S}(x)\rangle & =\frac{1}{(\pi x)^{2}}+B_{1} \frac{\cos \left(2 k_{F} x\right)}{x^{K_{\rho}+1}}
\end{aligned}
$$

where $K_{\rho}$ is a non-universal correlation exponent and $k_{F}$ is the Fermi momentum. Besides, local charge and spin perturbations, such as introduced by impurities or boundaries, lead to corresponding Friedel ossillations,

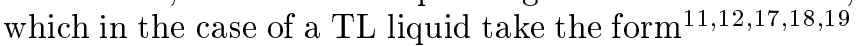

$$
\begin{aligned}
\langle\delta n(x)\rangle & =C_{1} \frac{\cos \left(2 k_{F} x\right)}{x^{\left(K_{\rho}+1\right) / 2}}+C_{2} \frac{\cos \left(4 k_{F} x\right)}{x^{2 K_{\rho}}} \\
\left\langle\delta S_{z}(x)\right\rangle & =D_{1} \frac{\cos \left(2 k_{F} x\right)}{x^{K_{\rho}}} .
\end{aligned}
$$

We first show our results for the total spin correlation function $c(j, k)=\left\langle\mathbf{S}_{j}^{T} \cdot \mathbf{S}_{k}^{T}\right\rangle$, where $\mathbf{S}_{j}^{T}=\mathbf{S}_{j}+\mathbf{s}_{j}$. Since 


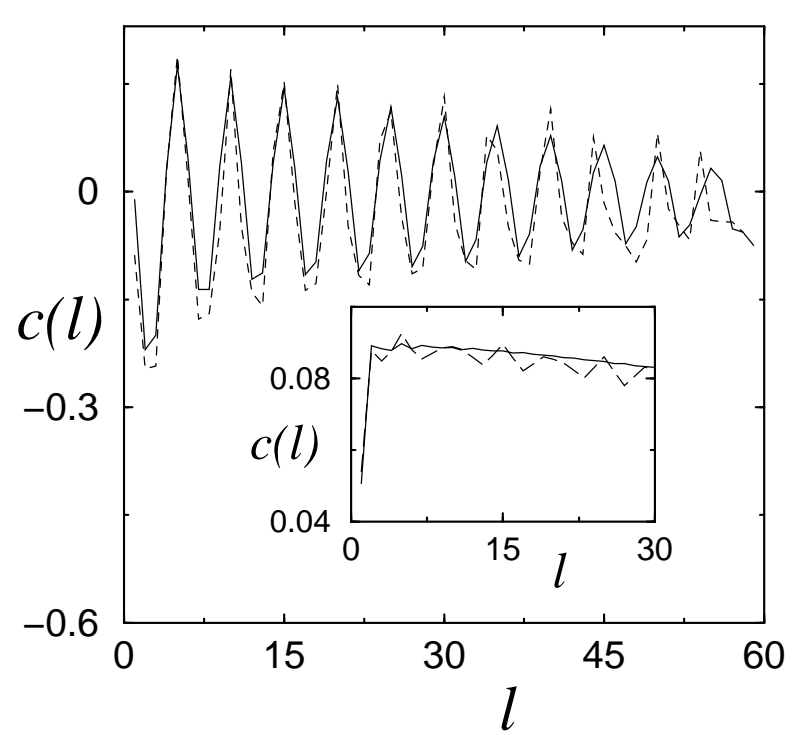

Figure 1: Spin-spin correlation function $c(l)$ for $n=\frac{2}{5}, L=$ 60 and $J=0.35$ (main) and $J=5$ (inset). The solid lines correspond to lattice averages, whereas the dashed lines are obtained with the two sites equidistant from the chain center (see text).

we use open boundary conditions, translational invariance is lost and $c(j, k)$ depends on both $j$ and $k$. We present results obtained by two different methods. In the first one (dashed lines in Fig. 1),$c(l=|j-k|)$ was obtained by taking $j$ and $k$ at the same distance (within a lattice spacing) from the center of the chain. We call this the central value of $c(l)$. In the second one (solid lines in Fig. 1), we averaged over all pairs of sites separated by the distance $l=|j-k|$. We will call it the average value of $c(l)$. If the boundary perturbation has a negligible effect on the spin-spin correlation function, then the two methods should yield similar results and we can have confidence that we have obtained the bulk value of $c(l)$. This is indeed what is observed for the density $n=\frac{2}{5}$ with $L=60$, as seen in Fig. 1. The paramagnetic (PM) and ferromagnetic (FM) phasest 15 can be easily discerned from the long distance behavior of $c(l)$ : for $J<J_{c}$, the envelope of $c(l)$ tends to zero (main plots in Fig. 1) and for $J>J_{c}$ it tends to the magnetization squared (inset of Fig. 1). The critical value is $J_{c} \approx 1.2$ for $n=\frac{2}{5}$. The ferromagnetism was also checked directly from the total spin of the ground state. The PM phase exhibits well developed Friedel oscillations due to the open boundaries.

In Fig. 2(a), we show the spin structure factor $S(q)=$ $\frac{1}{L} \sum_{j, k} e^{i q(j-k)} c(j, k)$ corresponding to Fig. 1. Because of the weak influence of the boundaries, this is very close to the Fourier transform of $c(l)$. Whereas $S(q)$ is maximum at $q=0$ in the FM phase (with $L S(0)=S^{T}\left(S^{T}+1\right)$ ) in the PM phase $S(q)$ is peaked at $q_{s}=\pi n$ at $n=\frac{2}{5} .4$ This result does not seem to be due to finite size effects. Indeed, the structure factor peak gets sharper and more pronounced as one goes from $L=60$ to $L=120$. This is shown in Fig. 2(b), for the two densities $n=\frac{2}{5}$ and $n=\frac{4}{5}$
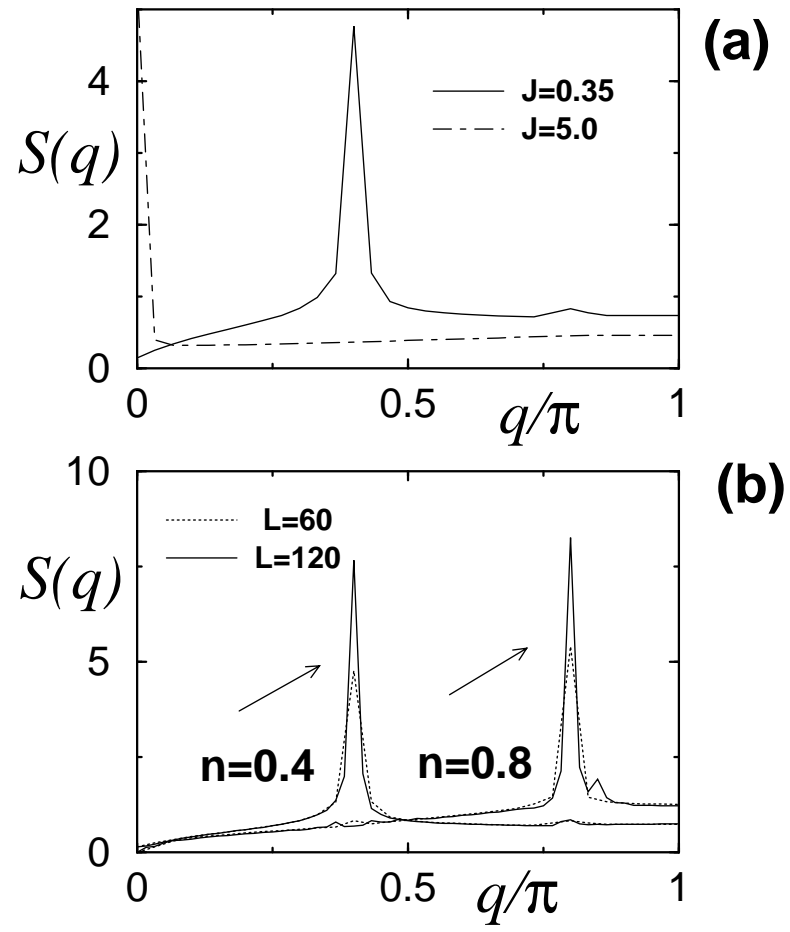

(b)

Figure 2: Spin structure factor $\mathrm{S}(\mathrm{q})$ : (a) $n=\frac{2}{5}$, same parameters as in Fig. 田; (b) $n=\frac{2}{5}$ and $n=\frac{4}{5}$ for both $L=60$ and $L=120$ (all at $J=0.35$ ).

at $J=0.35$. This is strong evidence for a small Fermi surface with $2 k_{F}=q_{s}=m_{m}$ in sharp contrast to what was previously reported 11.12 Note a very small feature at wave vector $2 \pi n$, which, however, does not increase with the system size and is below the accuracy of the DMRG. We also calculated the spin structure factor at several other density values, as shown in Fig. 3(a). As finite size effects appear to be negligible, we have kept to smaller chain sizes $(L=40$ or 30$)$. In all cases, there was good agreement between central and average values of $c(l)$. All the results point to the presence of a small FS. In order to understand the origin of this discrepancy we re-examined the parameter ranges studied in Refs. 11 and 12. As we will see, their results occur at large values of $J(J \gtrsim 1)$, where strong boundary charge perturbations mask the true bulk behavior of spin correlations. This is diagnosed by very different values of the average and the central $c(l)$. On the other hand, when $J \lesssim 1$ as in all cases seen above, the boundaries induce only a weak charge disturbance. As a result, central and average $c(l)$ are the same, and the spin correlation function oscillates at $2 k_{F}=q_{s}=\pi n$.

In Fig. 3(b), we present the local density $\left\langle n_{j}\right\rangle$ and the squared $z$-component of the total spin $\left\langle\left(S_{j z}^{T}\right)^{2}\right\rangle$ versus distance for the density $n=\frac{6}{7}$ and $J=2.5$. The charge Friedel oscillations induced by the-open boundaries are the same as found previously 11.2 and the squared $z$-component is anti-correlated with the charge. This strong charge disturbance is seen at other densities 


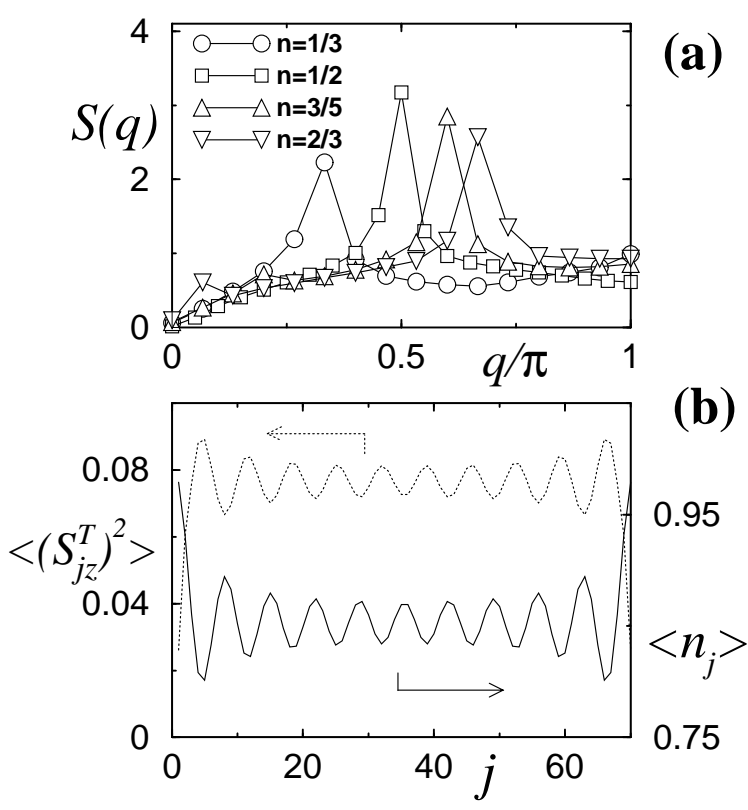

Figure 3: (a) $S(q)$ vs. momentum for several densities. In all cases, $J=0.5$ and $L=30$, except for $n=\frac{1}{2}$, where $L=40$. (b) $\left\langle\left(S_{j z}^{T}\right)^{2}\right\rangle$ and $\left\langle n_{j}\right\rangle$ vs. distance for $L=70, J=2.5$, and $n=\frac{6}{7}$. Peaks of $\left\langle\left(S_{j z}^{T}\right)^{2}\right\rangle$ track the valleys of $\left\langle n_{j}\right\rangle$.

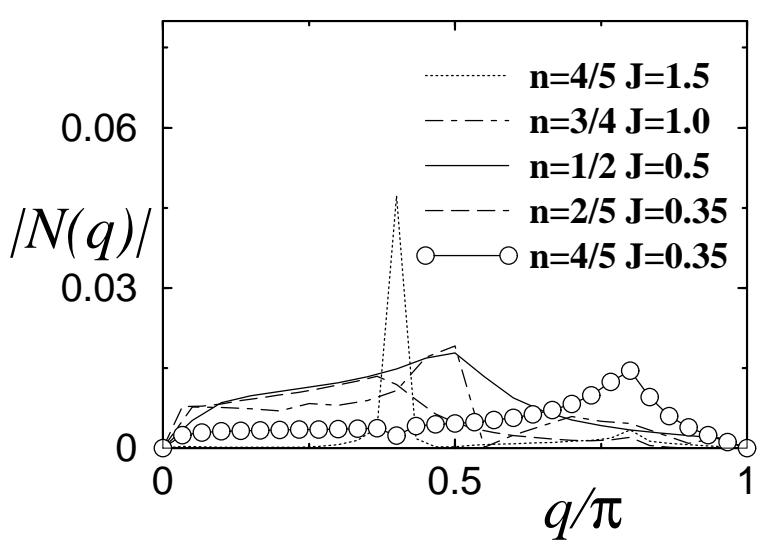

Figure 4: $|N(q)|$ vs. momentum for several densities. Coupling constants are indicated and lattice sizes are, from top to bottom, $L=60,40,40,60$, and 60 , respectively.

when $J \gtrsim 1$ but is suppressed as $J$ is decreased. This can be seen in Fig. 1 , where we show the magnitude of the Fourier transform $N(q)=\frac{1}{L} \sum_{j} e^{i q j}\left(\left\langle n_{j}\right\rangle-n\right)$ versus momentum for several densities and coupling constants. Furthermore, when $J$ is decreased, the charge Friedel oscillation peak moves from $q_{c}=2 \pi(1-n)$ to $q_{c}=2 k_{F}=\pi n$. We note that a peak at $q_{c}=2 \pi(1-n)$ $(\bmod 2 \pi)$ cannot distinguish between a small FS, with $4 k_{F}=2 \pi n$, and a large one, with $4 k_{F}^{*}=2 \pi(1+n)$ (we denote a large Fermi vector by a star). For this reason, we cannot use the charge structure factor to determine the size of the Fermi surface. Even when the peaks are not sharp, the oscillations are still quite well defined,
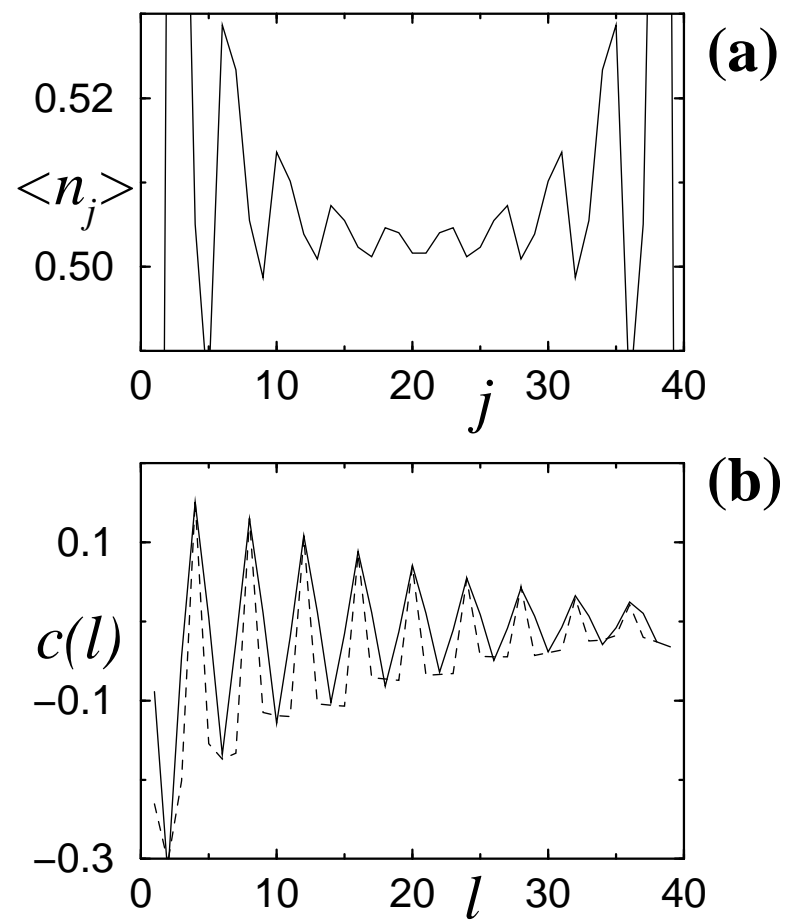

Figure 5: (a) $\left\langle n_{j}\right\rangle$ vs. distance for $L=40, J=0.5$ and $n=\frac{1}{2}$. (b) Spin-spin correlation function for $n=\frac{1}{2}$ with $J=0.5$ and $L=40$. Solid and dashed lines correspond to average and central $c(l)$, as in Fig. 1 .

as shown in Fig. 5 (a) for $n=\frac{1}{2}$ (compare the scales of Fig. 司(a) and Fig. $3(\mathrm{~b})$ ).

The presence of strong boundary charge disturbances leads to different behaviors of the central and the average spin correlation functions. This connection is made apparent in Figs. 5(b) and 6(a), which show average and central $c(l)$ for $n=\frac{1}{2}, J=0.5$ and $n=\frac{4}{5}, J=1.5$, respectively. At $n=\frac{1}{2}, J=0.5$, charge oscillations are small and the average and central $c(l)$ are almost the same. On the other hand, the large charge peak that occurs at $n=\frac{4}{5}, J=1.5$ (Fig. (4) leads to quite different values of the average and central $c(l)$. This is also reflected in the spin structure factor, which shows only broad ill-defined features, as plotted in Fig. 6(b). This is the key to understanding the discremancy between our results and the ones of Shibata et al.11.12

In order to observe spin oscillations, Shibata et. al applied a small local field at the chain ends and measured $\left\langle S_{j z}^{T}\right\rangle$. We obtained similar spin oscillations for $\left\langle\left(S_{j z}^{T}\right)^{2}\right\rangle$, but with half the period, without any field. The origin of the latter structure is clear: local spin fluctuations are determined by the charge Friedel oscillations (see Fig. 3(b)). Furthermore, this also shows that the system is rendered more polarizable by the boundary perturbation at the peaks of $\left\langle\left(S_{j z}^{T}\right)^{2}\right\rangle$, accounting for the oscillations of $\left\langle S_{j z}^{T}\right\rangle$ when a magnetic field is applied at the endpoints. Thus, the response of the system under 


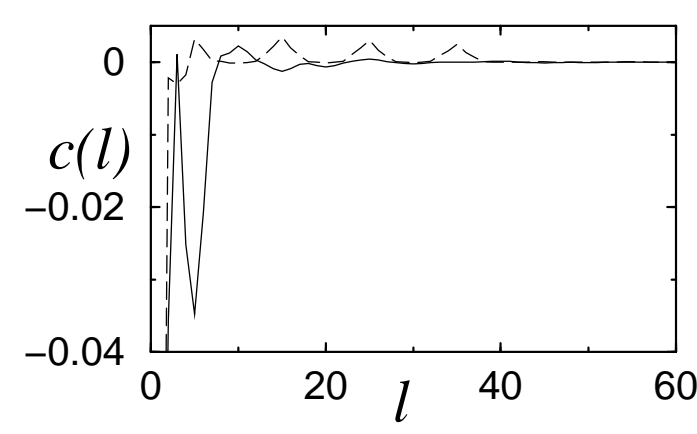

(a)

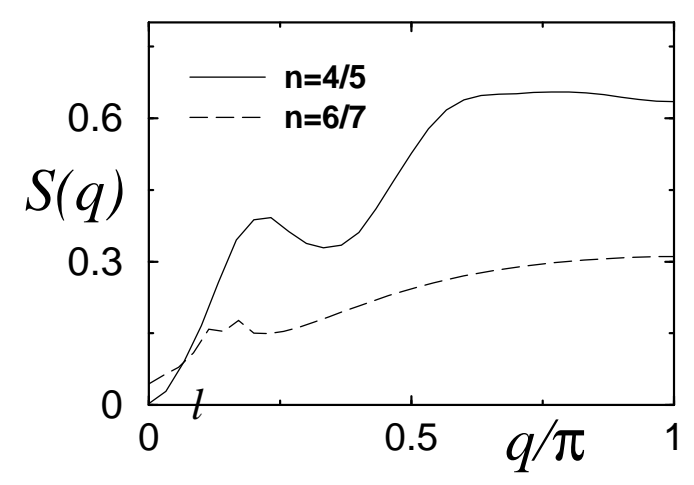

(b)

Figure 6: (a) Average (solid) and central (dashed) $c(l)$ for $n=\frac{4}{5}$ with $J=1.5$ and $L=60$, as in Fig. 1. (b) $S(q)$ vs. momentum. The densities are indicated. The parameters are the same as in Figs. 3 and 4 for these densities.

the application of boundary magnetic fields, as was done in Refs. 11 and 12, is strongly perturbed by the presence of open boundaries and cannot by itself be used to determine the size of the FS. In that case, the spin oscillations are inextricably linked to the charge ones by $q_{s}=q_{c} / 2$. Additional confidence in this picture can be gained by the inspection of Figs. 2 and 4 of Ref. 11, where an increase of the charge peak is accompanied by an increase of the spin peak. Spin Friedel oscillations should ideally be studied by applying a small local magnetic perturbation in the absence of any charge perturbation. We conclude that, based on the available evidence, it is impossible to determine whether the system has a large or a small Fermi surface for $J \gtrsim 1$.

We can envisage three alternatives to try to overcome this difficulty. The first one would be to use larger boundary fields so that the spin perturbation can surpass the charge one. However, it is not clear that this regime is attainable without drastically changing the nature of the ground state. A second way would be to work with periodic boundary conditions and a magnetic field applied at one site only, thus eliminating boundary charge perturbations while keeping spin ones. However, this is not particularly appealing because the DMRG loses accuracy with periodic boundary conditions. Finally, by going to larger systems one can have access to the true bulk behavior. Without these further studies, the size of the Fermi surface at $J \gtrsim 1$ remains undetermined.

We have also calculated the spin correlations between localized spins only and conduction electrons only. In

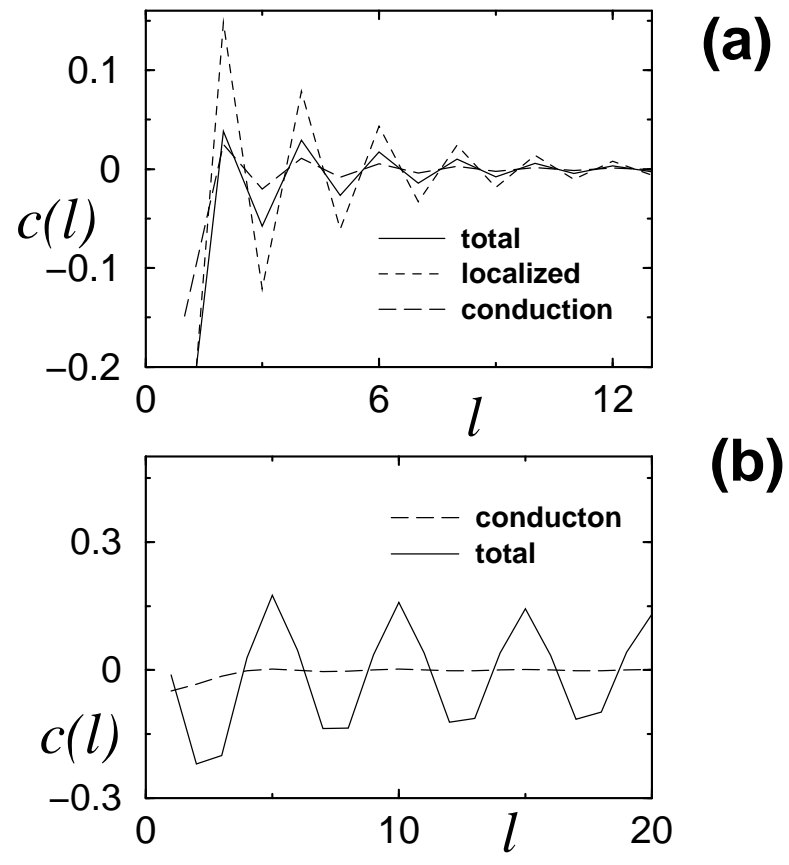

Figure 7: (a) The average spin-spin correlation function vs. distance for the total, localized and the conduction electron spins in a chain of $L=42$ sites, with $J=1.0$ at half filling. (b) Same as (a) but for a chain of $L=60$ sites, $J=0.35$, and $n=\frac{2}{5}$. Here, the correlations between localized spins are not shown. Both in (a) and (b), only the first few sites are shown.

Fig. đ(a) and (b) we present these correlations together with the total spin-spin correlations for densities $n=1$ and $n=\frac{1}{4}$, respectively. In Fig. G(b), the correlations between localized spins are not shown since they differ very little from the total spin ones. As expected all three functions have the same period, differing only in amplitude. It is also clear that the conduction electron contribution increases with the density. Note also that the spin correlations at half filling (Fig. Z(a)) decay much faster than at other fillings, due the presence of a spin gap.20.21

Let us now discuss these results. The theorem of Ref. 9 guarantees that, provided there is either unbroken time reversal or parity symmetries in the ground state, the one-dimensional Kondo lattice has gapless excitations at $2 k_{F}^{*}=\pi(1-n)$. This should be valid within the PM phase. The conventional Luttinger liquid phenomenology then tells us that these are collective spin and/or charge bosonic excitations with a linear dispersion with respect to deviations from this wave vector. They lead to the characteristic oscillatory behavior of Eqs. (1i) and (2) and the corresponding peaks in the spin and charge structure factors. Of course, the theorem does not forbid the appearance of gapless excitations at other wave vectors such as $2 k_{F}=\pi n$. Together with our results, this would seem to indicate that the spectral weight at $2 k_{F}^{*}$ is rather small in the range $J \lesssim 1$, most of it being concentrated at $2 k_{F}$.

The conventional wisdom about heavy fermion systems 
is based on the competition between the local Kondo effect and the inter-site Ruderman-Kittel-Kasuya-Yosida (RKKY) interaction. Although this dichotomy is controversial in one dimension, it is tempting to use it as a general guide. The size of the compensating Kondo cloud around a single localized moment has been argued to be exponentially large $a e^{1 / \rho J}$, where $a$ is the lattice spacing and $\rho$ is the density of states at the Fermi level 22 with typical values on the order of $1 \mu \mathrm{m}$. Thus, it would increase as $J$ is decreased towards the physically relevant region $J \lesssim 1$, where we observe the peak at $q_{s}=2 k_{F}=\pi n$. This might lead us to think that we should work with system sizes that are at least as large as the Kondo compensating cloud in order to observe features characteristic of a large FS. However, the fact that the peak at $2 k_{F}$ becomes sharper and more pronounced as $L$ is increased (Fig. 3(b)) casts doubt on this naive expectation. Moreover, even if the true thermodynamic limit of the spin correlations do indeed oscillate at $2 k_{F}^{*}=\pi(1+n)$, our results show that perhaps at the physically relevant distance scale the important wave vector is actually $2 k_{F}=\pi n$. For example, neutron scattering experiments are limited by the coherence length of the neutron beam and may not be able to probe large distances such as $a e^{1 / \rho J}$. Other probes of the FS size, such as quantum oscillations, are limited by the electron mean free path, which would also have to exceed this length scale to access the asymptotic limit. Thus, our results put stringent limits on the observability of a large FS in heavy fermion systems. Besides, the presence of disorder and/or inelastic scattering may render the small FS size the only relevant length scale in real systems.

In conclusion, we have found clear signatures of a small Fermi surface in the spin correlation function of the onedimensional Kondo lattice at small values of the Kondo coupling constant $(J \lesssim 1)$. The discrepancy with previous results in the literature that had argued for a large Fermi surface in this system is ascribed to the presence of large edge perturbations introduced by the use of open boundary conditions. These perturbations are larger for large coupling constants $(J \gtrsim 1)$, which hinders the investigation of the Fermi surface size in this region. Even if the true asymptotic spin correlations are peaked at the large Fermi surface wave vector, the relevant oscillation period in many cases of interest may be set by the size of the small Fermi surface.

JCX is grateful to A. L. Malvezzi for providing some DMRG data for comparison. We also thank I. Affleck for constructive criticism and suggestions. This work was supported by the Brazilian agencies FAPESP (00/028027 and 01/00719-8) and CNPq (301222/97-5).
* Electronic address: icxavier@ifi.unicamp.br

$\dagger$ Electronic address: peres@ifi.unicamp.br

¥ Electronic address: emiranda@ifi.unicamp.br

1 A. C. Hewson, The Kondo Problem to Heavy Fermions (Cambrige University Press, Cambridge, 1993).

2 P. Coleman, C. Pépin, Q. Si, and R. Ramazashvili, J. Phys.: Condens. Matter 13, 723 (2001).

3 J. A. Hertz, Phys. Rev. B 14, 1165 (1976).

4 A. J. Millis, Phys. Rev. B 48, 7183 (1993).

${ }^{5}$ Q. Si, S. Rabello, K. Ingersent, and J. L. Smith, Nature 413, 804 (2001).

${ }^{6}$ R. M. Martin, Phys. Rev. Lett. 48, 362 (1982).

7 J. Friedel, Philos. Mag. 43, 153 (1952).

8 J. M. Luttinger, Phys. Rev. 119, 1153 (1960).

9 M. Yamanaka, M. Oshikawa, and I. Affleck, Phys. Rev. Lett. 79, 1110 (1997).

10 M. Oshikawa, Phys. Rev. Lett. 84, 3370 (2000).

11 N. Shibata, K. Ueda, T. Nishino, and C. Ishii, Phys. Rev. B 54, 13495 (1996).

12 N. Shibata, A. Tsvelik, and K. Ueda, Phys. Rev. B 56, 330 (1997).
13 S. R. White, Phys. Rev. Lett. 69, 2863 (1992).

14 S. R. White, Phys. Rev. B 48, 10345 (1993).

15 H. Tsunetsugu, M. Sigrist, and K. Ueda, Rev. Mod. Phys. 69, 809 (1997).

16 J. Voit, Rep. Prog. Phys. 57, 977 (1994).

17 S. Eggert and I. Affleck, Phys. Rev. Lett. 75, 934 (1995).

18 R. Egger and H. Grabert, Phys. Rev. Lett. 75, 3505 (1995).

19 J. Voit, Y. Wang, and M. Grioni, Phys. Rev. B 61, 7930 (2000).

${ }^{20}$ H. Tsunetsugu, Y. Hatsugai, K. Ueda, and M. Sigrist, Phys. Rev. B 46, 3175 (1992).

21 C. C. Yu and S. R. White, Phys. Rev. Lett. 71, 3866 (1993).

22 E. S. Sørensen and I. Affleck, Phys. Rev. B 53, 9153 (1996).

23 We adhere to the somewhat inappropriate yet common usage of the term Fermi "surface" in one dimension.

24 The residual value of $S(q=0)$ in the PM phase is due to the DMRG truncation at $m=400$. We have checked that it decays exponentially to zero as $m$ is increased. 